\title{
Application of Nanofluid Injection for Enhanced Oil Recovery (EOR)
}

\author{
Abdelrahman El-Diasty*, Hamid Khattab, Mahmoud Tantawy \\ Faculty of Petroleum and Mining Engineering, Suez University, Egypt \\ *abdelrahman.eldiasty@gmail.com
}

\begin{abstract}
The use of nanofluids has been investigated and established for several applications in the oil and gas industry. Using nanoparticles for Enhanced Oil Recovery (EOR) applications underlines their small size in comparison with the size of the rock pore throats; consequently, they could easily transport into porous rocks with minimum retention effect and permeability reduction. Nanoparticles can significantly increase the oil recovery by enhancing both the fluid properties and fluid-rock interaction properties. In this study, commercial silica nanoparticles dispersions were used in standard core flooding experiments to evaluate the effect of the nanofluid injection on the incremental oil recovery. This will open the door for taking the nanotechnology from the lab to the oil field.
\end{abstract}

Keywords: Enhanced Oil Recovery (EOR), Nanotechnology, Nanofluids, Nanoparticles, Surface Area, Porous Media.

\section{Introduction}

The oil field recovery cycle is divided into three main stages: primary recovery, secondary recovery, and tertiary recovery. In the primary recovery stage, the oil production results from the displacement energy that is naturally present in the reservoir, such as gas-cap drive, solution-gas drive, and natural water drive, etc. After reaching a certain limit of production performance, a supplemental energy is needed to support the reservoir to keep producing. This is where the secondary recovery stage starts. The secondary recovery processes are mainly waterflooding and gas injection. Then it comes the tertiary recovery (Enhanced Oil Recovery "EOR") stage, using some processes to alter the fluid-rock system and enhance the oil recovery.

Nanotechnology is defined as the application of very small pieces of material(s) with dimensions between approximately 1 and 100 nanometers. Using nanoparticles can drastically increase the oil recovery by improving both the injected fluid properties and fluid-rock interaction properties. The injected fluid can have enhanced viscosity, enhanced density, reduced surface tension, and improved emulsification. The fluid-rock interaction properties mainly incorporate the wettability alteration. 


\section{Applications of Nanoparticles in EOR Processes}

Exploiting the distinct properties and unique phenomena of nanoparticles, at that scale ranging from individual atoms or molecules to about 100 nanometers as compared to those associated with bulk behavior, novel real applications of materials, devices and systems have been available in many industries.

The nanoparticles present many advantageous characteristics such as high surface area, active surfaces, and special optical and chemical responses. The nanoparticles have a very large specific surface area, which increases exponentially with the decrease in the diameter of the particles.

\subsection{Nanofluids}

A nanofluid is simply defined as a colloidal suspension of nanoparticles with an average size of 1 to $100 \mathrm{~nm}$. The mechanisms of nanofluid EOR have been investigated in many previous studies, which mainly include disjoining pressure, temporary pore channels plugging (log-jamming), increased viscosity of injection fluids (decreasing the mobility ration), IFT reduction, and wettability alteration.

\subsection{Disjoining Pressure}

The nanoparticles present in the nanofluids tend to form a film that takes the shape of a wedge on contact with oil phase. This wedge-like film acts to separate the oil droplets from the rock surface, in that way recovering more oil than previously possible with using conventional injection fluids. The main drive to form this wedge-shaped film is the existence of a pressure called "structural disjoining pressure" [1], [2], [3].

The injection pressure of nanofluids drives the injected nanofluids to apply a pressure forcing the nanoparticles in the interface region forward, and the particles tend to structurally arrange themselves in self-assembled layers. The result of this arrangement applies additional disjoining pressure at the confined interface region more than that in the bulk liquid. This can be better illustrated below in Figure 1 by the shape of the interface profile in the wedge region in both cases where the nanoparticles are present and absentError! Reference source not found..

During the nanofluid propagation in the porous media, there are some mechanisms that could potentially cause a reduction in the dispersed nanoparticles concentration [4]. The main reason for the nanoparticles retention in the porous media is adhering on the pore walls and blocking of pore throats. The blocking of pore throats could be caused by two main mechanisms: Mechanical entrapment (size exclusion) and Log-jamming (accumulation) [5]. 


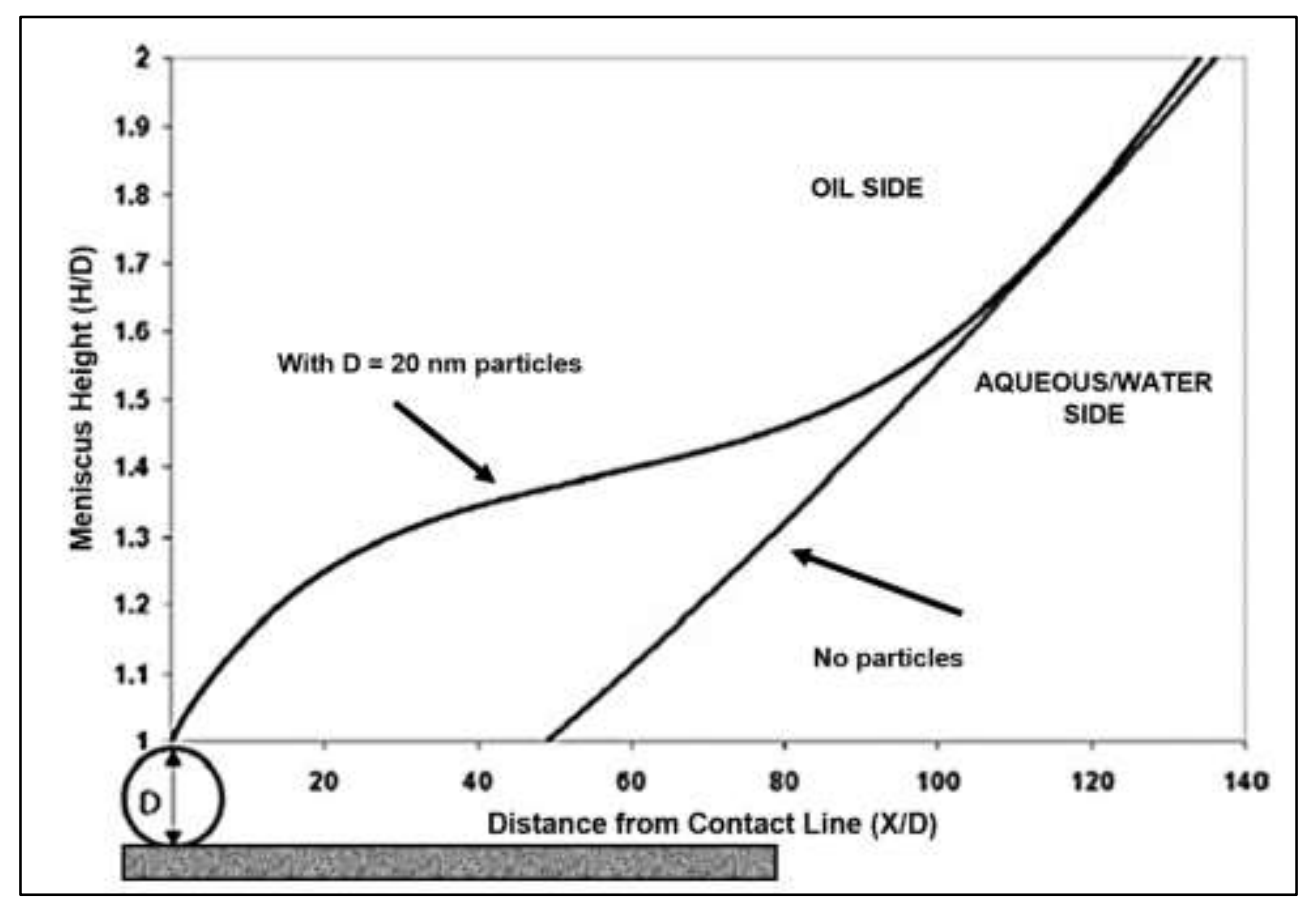

Figure 1. Shape of the Interface Profile in the Confined Wedge Region (After [3])

\subsection{Pore Channels Log Jamming}

Mechanical entrapment is defined as the blockage of the pore throats when the nanoparticle has a larger diameter than the pore throats it is blocking. Therefore, the nanoparticles are not able to enter the pore channel, i.e., blocking due to particle diameter that is larger than the pore throat diameter, as illustrated below in Figure 2Error! Reference source not found..

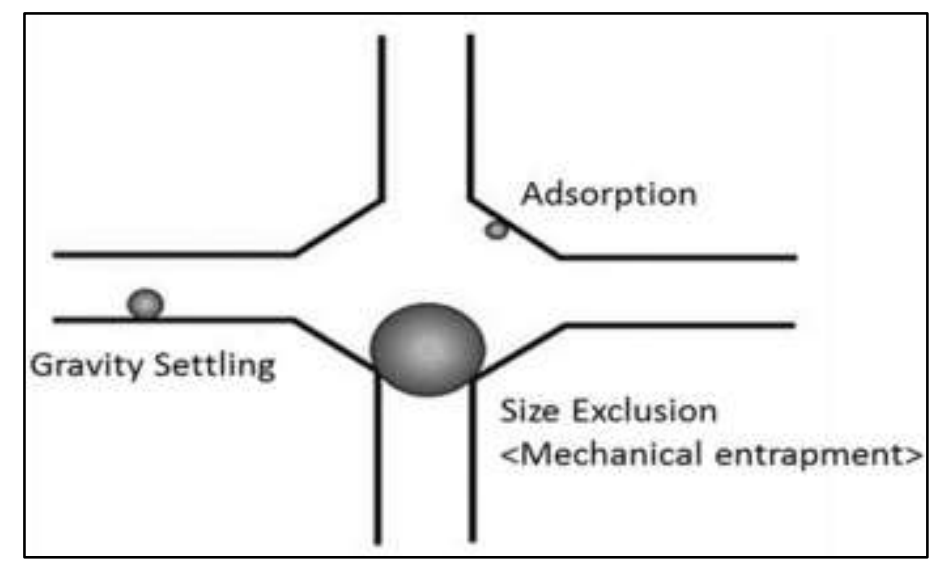

Figure 2. Particle Retention Mechanisms

To avoid this mechanical entrapment during the nanofluid flooding, the diameter of the nanoparticles has to be much smaller than the diameter of the pore throat, which is the actual case for the nanofluid flooding where reservoir rock 
pores are in the scale of micrometers, thousand times bigger than the nanometer scale of nanoparticles.

Log-jamming is defined as the blockage of pore throats even when they are much larger than each individual nanoparticle. Thinking of the pore throats as traffic jams in the pore channel, and because of the narrowing of the flow area open to the nanofluid and the constant pressure difference, the velocity of the fluid will increase at the pore throat. The carrying fluid molecules, water $\left(\mathrm{H}_{2} \mathrm{O}\right)$, will eventually accelerate much faster than the nanoparticles which will lead to the accumulation of nanoparticles at the entrance of the pore throats [5]. At the beginning, the diameter of these throats will be reduced step by step then the blockage occurs, as illustrated below in Figure 3Error! Reference source not found..

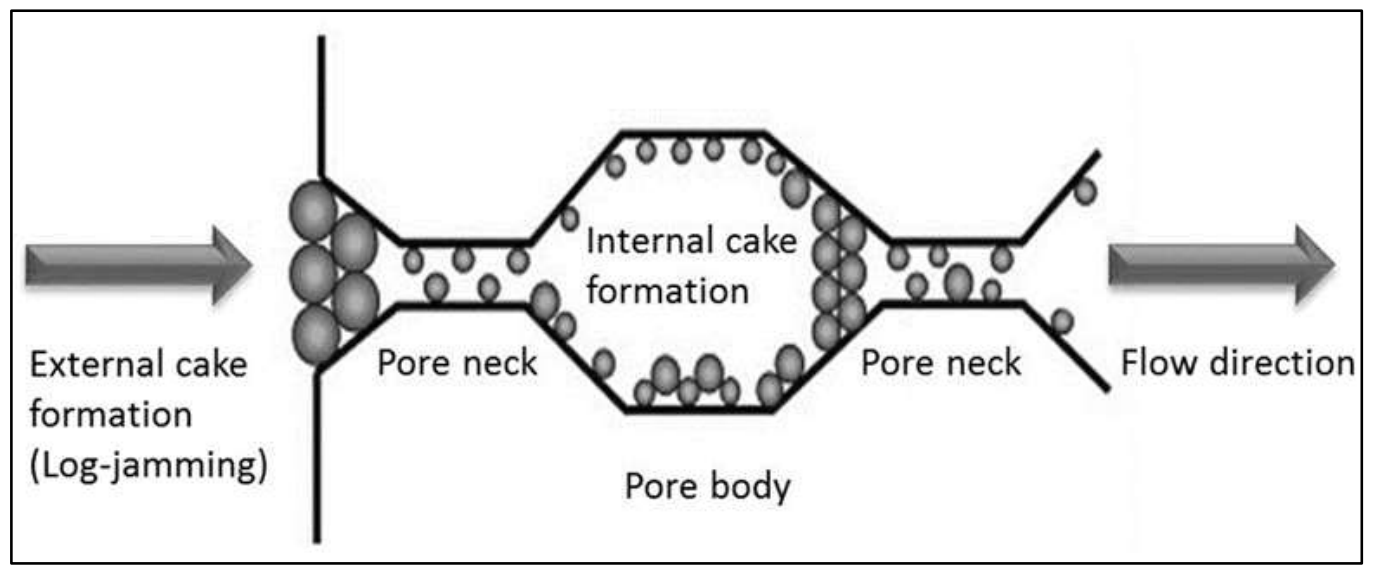

Figure 3. Log-Jamming - Accumulation of Particles

The blockage of the pore throats due to the log-jamming can be beneficial. In the extremely small pore throats, because of the density difference between the nanoparticles and the carrying fluid (water), the nanoparticles movement might slow down and accumulate at the throats. As illustrated in Figure 4, the pressure starts to build up in the adjacent pores, which results to forcing out the trapped oil droplets. Then once the oil droplet is moved, the adjacent pressure consequently drops, and consequently the blockage occurring at throat gradually dissolves, and the particles commence flowing with the water. This temporary log-jamming mechanism is mainly controlled by the concentration, nanoparticles size, injection rate, and the diameter of the pore throats (the pore size distribution). 


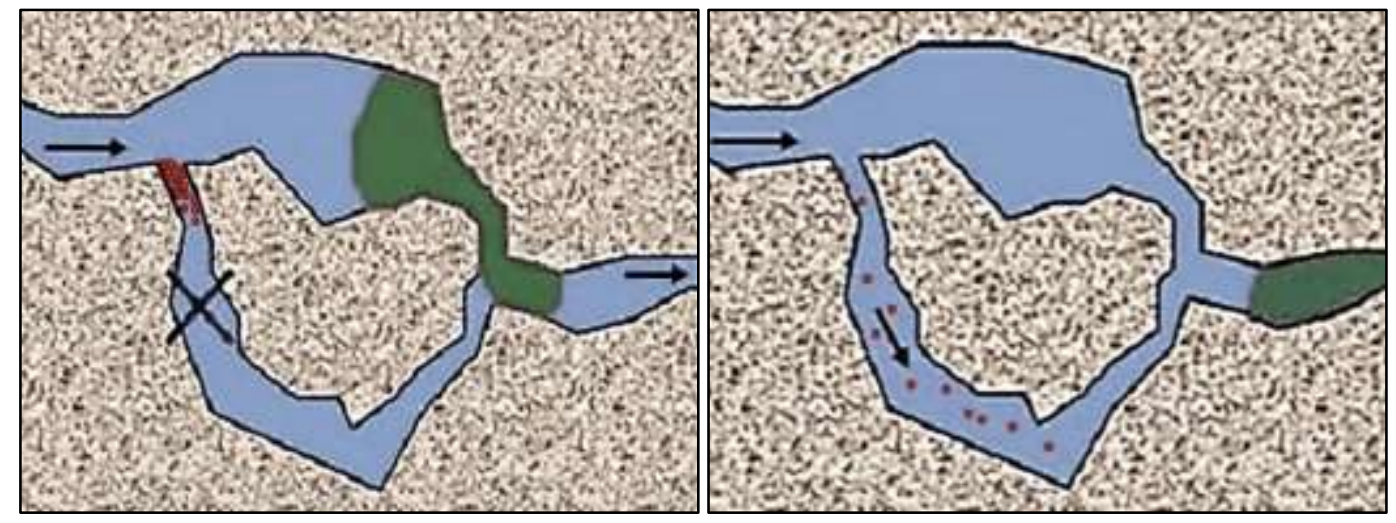

Figure 4. Illustration of the Temporary Log-Jamming

\subsection{Decreasing the Mobility Ratio}

One of the most important parameters that affect the EOR processes is the mobility ratio, which is defined as the mobility of an injectant, such as the nanofluid, divided by the mobility of the fluid being displaced, such as oil. Where the mobility itself is defined as the ratio of effective permeability to phase viscosity. The viscosity of nanofluids eventually increases with increasing the nanoparticles concentration and the carrying fluid "brine" salinity. The type of nanoparticles can also affect the viscosity of the nanofluid. For instance, at a certain concentration, the viscosity of the $\mathrm{SiO} 2$ nanofluid is measured to be higher than the viscosity of the $\mathrm{Al} 2 \mathrm{O} 3$ nanofluid [6].

\subsection{IFT Reduction}

Many experiments were conducted to study how the IFT values change between the crude oil and a variety of metal nanofluids. [7] investigated the effects of $\mathrm{SiO}_{2}$ and $\mathrm{Al}_{2} \mathrm{O}_{3}$ nanofluids on IFT measured at the ambient pressure and temperature. They concluded that the IFT clearly decreased when either $\mathrm{SiO}_{2}$ or $\mathrm{Al}_{2} \mathrm{O}_{3}$ nanoparticles were added into brine. Though, the $\mathrm{SiO}_{2}$ nanofluid had a lower IFT value than the $\mathrm{Al}_{2} \mathrm{O}_{3}$ nanofluid.

Moreover, [8] further studied the ability of three different nanoparticles $\left(\mathrm{SiO}_{2}, \mathrm{Al}_{2} \mathrm{O}_{3}\right.$, and $\left.\mathrm{NiO}\right)$ to reduce IFT between the crude oil and nanofluids. They came to the conclusion that the $\mathrm{SiO}_{2}$ nanoparticles resulted in the lowest IFT value, while the $\mathrm{NiO}$ nanoparticles showed the least reduction on IFT. The main justification for the IFT reduction is referred to the high adsorption of nanoparticles that modifies oil and water surface.

\subsection{Wettability Alteration}

Several studies were conducted to experimentally investigate the effect of various nanofluids on the reservoir rock wettability alteration.

[9] concluded that $\mathrm{SiO} 2$ nanoparticles can change the wettability of oil-wet sandstone cores to neutral-wet. As for carbonates, [10] concluded that $\mathrm{SiO} 2$ 
nanoparticles could be used as wettability modifiers for carbonate cores as well. In addition, [11] suggested that the wettability of the shale can be altered from oil-wet to water-wet using nano-biomaterials.

In point of fact, there are certain factors, such as nanoparticles concentration, nanoparticles size, and water salinity that can affect the wettability alteration caused by nanofluids. [9] found that water wetness of the treated rock increased with increasing the nanoparticles concentration. Moreover, [12] studied the influence of the nanoparticles size on wettability alteration. They reported that the contact angle decreased as nanoparticles size decreased. When we have a large number of nanoparticles and smaller size, thus the electrostatic repulsion force between the nanoparticles is bigger.

\section{Research Design and Methodology}

In this project, the ultimate target was to understand the mechanisms behind the additional recovered oil with using nanoparticles as an EOR method (nanofluid flooding), and to understand how to optimize the nanoparticles concentration required for the highest recovery. The experimental work of the project started with preparing the core samples to simulate the actual reservoir rock conditions. A number of 1 " and 1.5" plugs were cut using a plugging machine then they were trimmed and had end-face grinding. After getting the core plugs into the required dimensions, a Soxhlet distillation extraction device was used to remove the liquid phases (oil and/or water) from the core. Once the samples were cleaned, the samples were left to dry in a conventional oven. After drying the samples, full measurements of weight and dimensions were taken down, then the Helium gas expansion Porosimeter was used to determine the samples grain and pore volume.

After measuring the porosity of the samples, they were fully saturated with formation water. Brine with $75,000 \mathrm{PPM} / \mathrm{NaCl}$ was used to saturate the samples. After saturating the samples with $100 \%$ of the water with the designed salinity, the samples weight was taken down to double check the porosity measured with the Helium Porosimeter which is usually a bit higher than the value driven by the difference between dry and wet weight due to the disconnected pores. Then the relative flooding apparatus was used to measure the absolute permeability under reservoir-representative confining pressure. After measuring the absolute permeability, the injection fluid was switched to oil. The flooding started at 0.2 $\mathrm{cc} / \mathrm{min}$ rate until no more water is getting out of the sample, then the injection rate was increased gradually while monitoring the pressure at each step until it stabilizes, and no more water gets out. This stage stays up to 8 hours to guarantee reaching the irreducible water saturation. The whole flooding process is performed with 2000 psi confining pressure to simulate the overburden pressure in the reservoir rock.

By then, using the porosity measurements and the volume of water out of the oil flooding, the Swi and So were calculated for each sample. At this stage, the sample was ready for investigating any secondary or tertiary recovery method, which is in this project, the nanofluid flooding. 


\section{Results Insights and Discussions}

Commercial $\mathrm{SiO}_{2}$ nanofluid was used in this project that has no coating applied on the surface of the particles. A little dispersant (1-2\% poly (Nvinylpyrrolidone) PVP) was applied to help disperse the nanoparticles and prevent the agglomeration. This nanofluid system could be applied economically in the oil field, given the ease of preparation, and the low cost, in comparison with most of the other nanofluids used in the literature. PVP was preferred rather than Polyethylene Glycol (PEG), Propanediol, and Propanol, due to the higher cost of the coating.

Proposing the idea of commercial nanofluid EOR method, we tried to study the effect of continuous nanofluid, 5\% wt. nanoparticles concentration, after the waterflooding breakthrough. The properties of the core sample used in this step are summarized in Table 1.

Table 1. Rock Properties of Samples Set \#1

\begin{tabular}{|l|c|}
\hline Sample number & X2 \\
\hline Sample type & Sandstone \\
\hline Diameter / Length $(\mathbf{m m})$ & $25.14 / 70.90$ \\
\hline Surface $\left.\mathbf{( c m}^{\mathbf{2}}\right)$ & 4.96 \\
\hline Bulk volume (cc) & 35.19 \\
\hline Grain density (g/cc) & 2.67 \\
\hline Mdry / Mwetted (g) & $68.47 / 78.69$ \\
\hline Pore Volume (cc) & 9.51 \\
\hline Porosity (\%) & $27.02 \%$ \\
\hline Permeability (mD) & 20.74 \\
\hline Swi $(\%)$ & $25.34 \%$ \\
\hline Experiment & Cont. 5\% LS NF \\
\hline
\end{tabular}

The results of the 5\% wt. nanofluid, illustrated in Figure 5, show increased oil recovery. However, there is an increase in the pressure, which implies reduction in the permeability due to the retention of the nanoparticles and the temporary plugging of the pore throats.

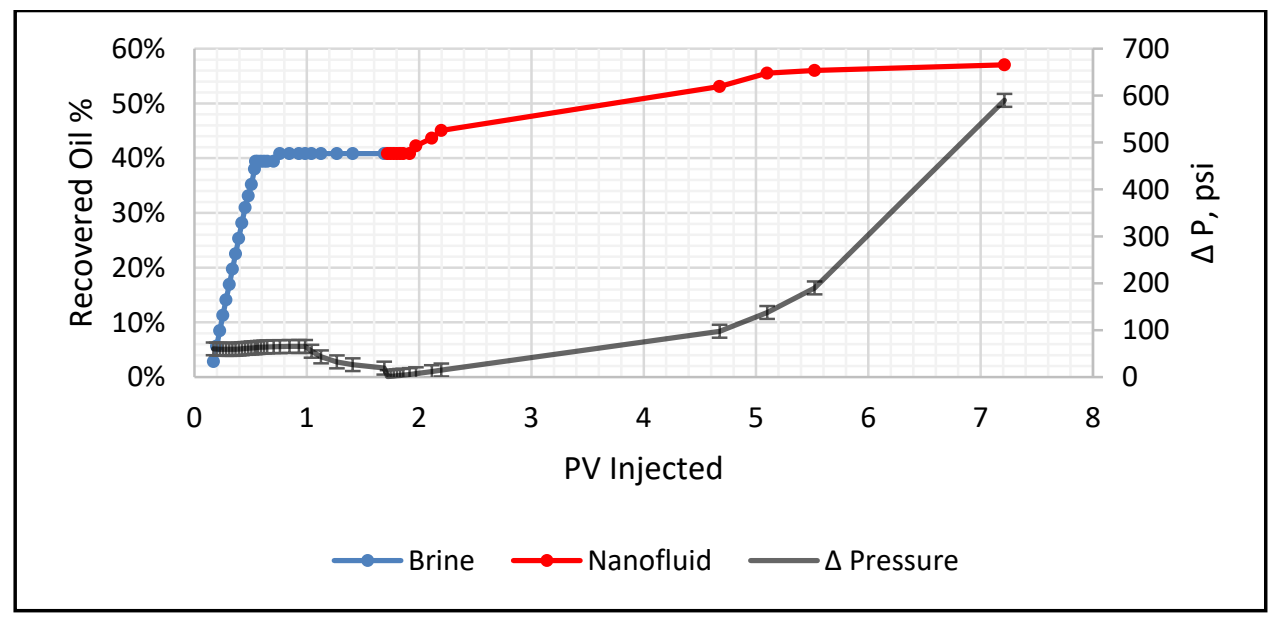

Figure 5. Injection of 5\% Wt. Nanofluid 
To investigate the retention of the nanoparticle in the porous media, a clean sample of the rock before any flooding and another sample after the nanofluid flooding were scanned by SEM, as shown in Figure 6, to compare the effect of the nanofluid flooding on the pore system.

In Figure 6 (b), we can see the agglomeration of the nanoparticles after the $5 \%$ wt. nanofluid flooding compared with the clean structure in Figure 6 (a). The pore throats have been blocked with these agglomerations. Hence, this system with such high particles concentration will not be applicable in the field on the reservoir scale as it will require very high injection pressure that might damage the reservoir if it exceeds the formation fracture pressure.
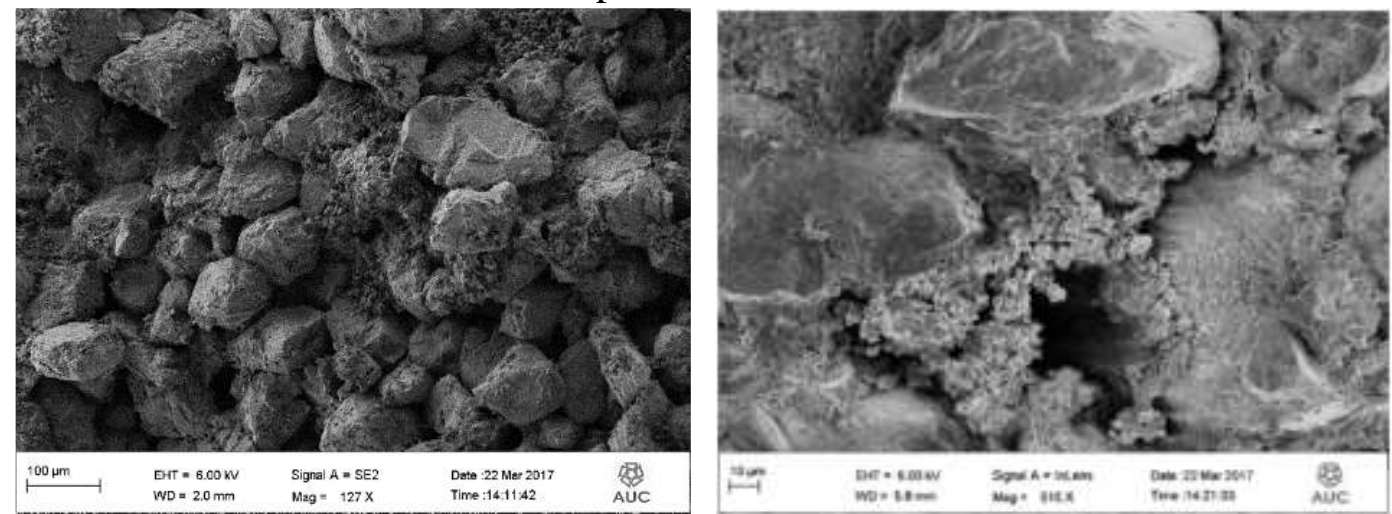

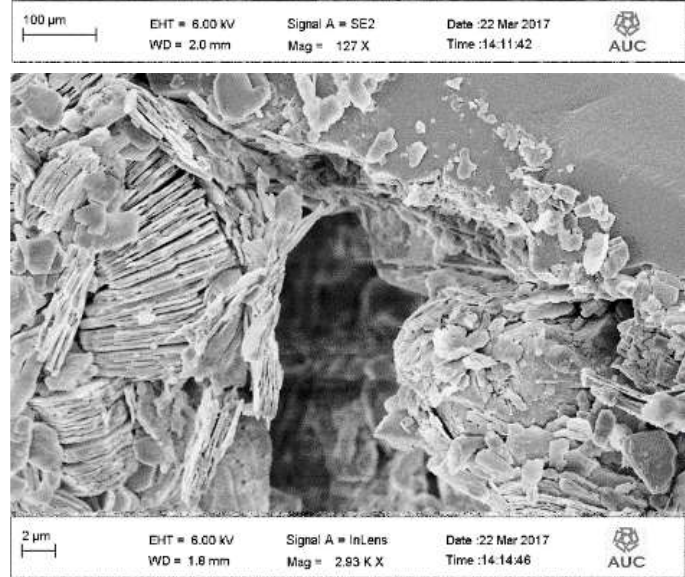

(a)

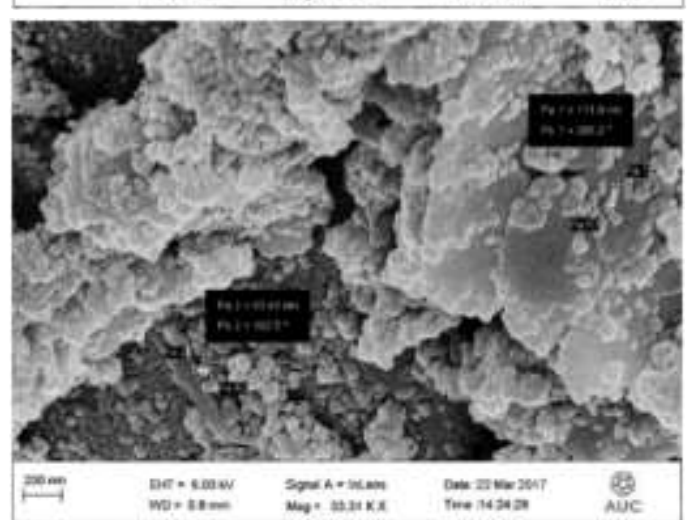

(b)

Figure 6. (a) Clean Rock Sample Before Flooding (b) After The 5\% Wt. Concentration Nanofluid Flooding

The above experiments were performed with injection rate of $0.2 \mathrm{CC} / \mathrm{min}$, which reflects the optimum "rule-of-thumb" flood front rate of $1 \mathrm{ft} / \mathrm{day}$.

\section{Conclusions}

These results propose a theory that there is a critical concentration of nanoparticles after which the recovered oil will decrease due to the agglomeration and retention of the particles in the pore system. This critical concentration equals the amount that will nearly $100 \%$ cover the surface area of the rock grains. As any extra particles will agglomerate in the pore throats and negatively affect the 
recovery and reduce the rock permeability. Hence, the main factor in the flooding process is the nanofluid system, whose design consists of particles size, particles concentration, and chemical modification.

The concentration "number of particles" can be estimated by dividing the volume of nanoparticles in one liter by the size of one nanoparticle, assuming spherical particle with density similar to silica density of $2.66 \mathrm{~g} / \mathrm{cc}$, Equation 1.

$C_{N P}=\frac{\frac{\rho_{\text {dispersion }} \times 1 L \times w t . \%}{\rho_{N P}}}{\frac{4 \pi}{3} r_{N P}^{3}} \quad \# / L_{\text {Conc. }}$

If volume $\mathrm{V}$ of nanoparticles trapped in pore space, assuming spherical particles with equal diameter touching each other at one-point, specific area can be defined as Equation 2.

$S_{b}=\frac{A}{V}=\frac{d^{2} \pi}{\frac{1}{6} d^{3} \pi}=\frac{6}{d}$

If we further assume $\mathrm{V}$ is the volume of the particles that will be adsorbed on the pore walls/surfaces, then we assume that $V^{-}$is the volume of particles that are going to be entrapped at pore throats, all normalized per unit bulk volume of the media. Moreover, we assume that the adsorption will happen as a single layer, then the total surface area that will be in contact with fluids, also normalized per bulk volume of porous media, is calculated by Equation 3.

$S=\beta\left(V-V^{\prime}\right) \times S_{b}$

Where $\beta$ is assumed to be the surface area coefficient. There are several methods to calculate the specific area of a sand core, but the most straightforward method would be by the following empirical formula [4], Equation 4.

$S_{v}=7000 \emptyset \sqrt{\frac{\emptyset}{K}}$

As illustrated below in Figure 7, the red layer represents the specific area of the particles "S", and the black layer represents the specific area of the rock grains "Sv".

At the time when $\mathrm{S}=\mathrm{Sv}$, it means that the total surface should be completely covered by particles adsorbed on the pore surfaces. At this stage, the wettability is determined by nanoparticle properties more than the rock surface itself. However, with more nanofluid injected where $\mathrm{S}>\mathrm{Sv}$, the additional deposition of particles will only negatively lead to reduction in porosity and permeability. 


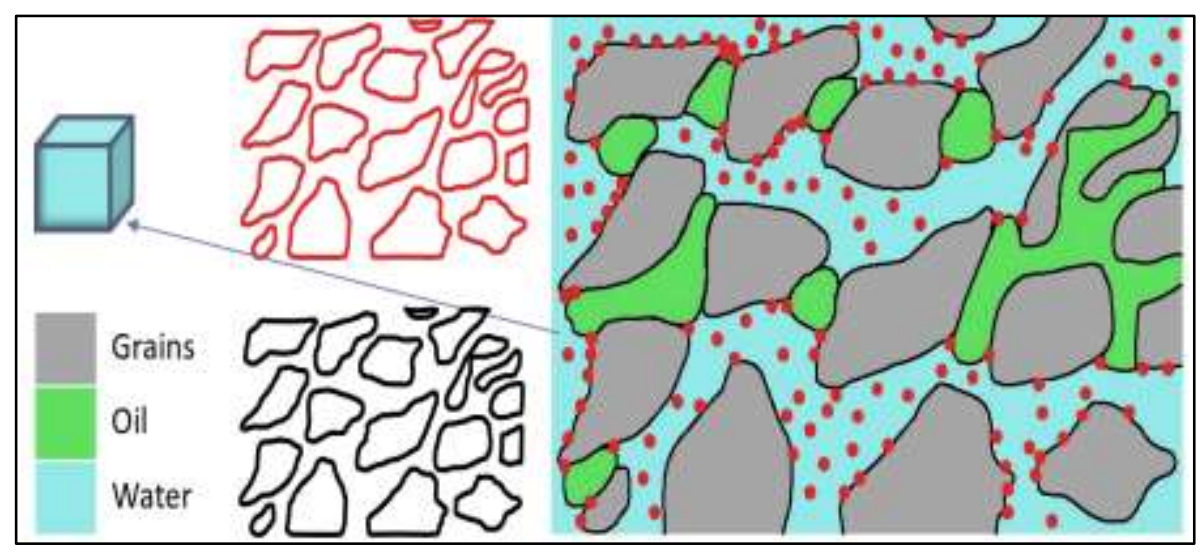

Figure 7. Illustration of Nanofluid Critical Volume in Unit Bulk Volume of the Pore System

The amount of the particles that will be adsorbed on the rock surface may change the wettability of the rock. Adhering and detaching of the particle on pore walls is mainly controlled by the Van der Waals attraction forces between the silica particle and the pore wall.

The main takeaways of the study could be summarized in the below points:

- The commercial nanofluid can be used as EOR method and achieve incremental recovery.

- There is a critical concentration of nanoparticles after which the recovered oil will decrease due to the agglomeration and retention of the particles in the pore system.

- The critical concentration equals the amount that will $100 \%$ cover the surface area of the rock grains. As any extra concentration will agglomerate in the pore throats and negatively affect the recovery and reduce the permeability.

- Optimizing the slug size and concentration of the nanofluid plays an important role in achieving the highest recovery factor.

\section{References}

[1] P. M. Mcelfresh, C. Olguin and D. Ector, "The Application of Nanoparticle Dispersions To Remove Paraffin and Polymer Filter Cake Damage," 2012.

[2] R. Aveyard, B. P. Binks and J. H. Clint, "Emulsions stabilised solely by colloidal particles," Advances in Colloid and Interface Science, Vols. 100-102, pp. 503-546, 2003.

[3] A. Chengara, A. D. Nikolov, D. T. Wasan, A. Trokhymchuk and D. Henderson, "Spreading of nanofluids driven by the structural disjoining pressure gradient," Journal of colloid and interface science, vol. 280(1), pp. 192-20, 2004.

[4] F. Caldelas, M. J. Murphy, C. Huh and a. S. L. Bryant, "Factors Governing Distance Of 
Nanoparticle Propagation In Porous Media," 2011.

[5] T. Skauge, S. Hetland, K. Spildo and A. Skauge, "Nano-Sized Particles For EOR," 2010.

[6] A. M. Ragab and A. E. Hannora, "An Experimental Investigation of Silica Nano Particles for Enhanced Oil Recovery Applications, " 2015.

[7] A. M. Salem and A. E. Hannora, "A Comparative Investigation of Nano Particle Effects for Improved Oil Recovery - Experimental Work," 2015.

[8] O. Alomair, K. Matar and Y. Alsaeed, "Nanofluids Application for Heavy Oil Recovery," in SPE Asia Pacific Oil \& Gas Conference and Exhibition, 2014.

[9] S. Li, M. Genys, K. Wang and O. Torsæter, "Experimental Study of Wettability Alteration during Nanofluid Enhanced Oil Recovery Process and Its Effect on Oil Recovery," 2015.

[10] A. Roustaei and H. Bagherzadeh, "Experimental investigation of SiO2 nanoparticles on enhanced oil recovery of carbonate reservoirs," Journal of Petroleum Exploration and Production Technology, vol. 5, no. 1, pp. 27-33, 2015.

[11] M. Mohebbifar, M. Ghazanfari and M. Vossoughi, "Experimental Investigation of Nano-Biomaterial Applications for Heavy Oil Recovery in Shaly Porous Models: A Pore-Level Study," Journal of Energy Resources Technology, vol. 137, no. 1, 2014.

[12] L. Hendraningrat, S. Li and O. Torsater, "Effect of Some Parameters Influencing Enhanced Oil Recovery Process using Silica Nanoparticles: An Experimental Investigation.," 2013.

[13] X. Sun, Y. Zhang, G. Chen and a. Z. Gai, "Application of Nanoparticles in Enhanced Oil: A Critical Review of Recent Progress," Energies, vol. 10, no. 3, p. 345, 2017.

[14] L. Hendraningrat, S. Li and O. Torsæter, "A coreflood investigation of nanofluid enhanced oil recovery," Journal of Petroleum Science and Engineering, vol. 111, pp. 128-138, 2013. 